\title{
Numeric comparison and integration: Alternative modes of processing
}

\author{
KENT L. NORMAN \\ University of Maryland, College Park, Maryland 20742
}

\begin{abstract}
The processes of numeric comparison and integration were investigated in a choice reaction time task requiring subjects to choose the pair of digits having the greatest total. Two alternative modes of processing were identified. Subjects either added each pair separately and then compared the totals or compared adjacent digits and then combined their differences. In one condition, the pairs of single digits were presented simultaneously. In two other conditions, two of the four digits were presented during the foreperiod in order to investigate the effect of sequential presentation on the selection of processing mode. In all conditions response time was found to be an inverse function of the split in total magnitude between the left and right pairs, as well as within- and between-pair orders of the digits. The results were interpreted in terms of a numeric processing model with comparison and integration stages. These stages conform to the empirical results obtained in other studies on single digits.
\end{abstract}

Many information processing tasks involve identifiably separate stages that are necessarily sequential but not necessarily of fixed order. When such stages are identified, two empirical questions are important. First, is there a naturally preferred order of processing; second, is it possible experimentally to alter the order of processing?

In this study subjects shown two pairs of singledigit numbers were asked to choose the pair having the greater total. The task inherently requires two operations which may be applied in either order. In the integration-comparison mode, the subject finds the total for each pair and then compares the totals to select the larger. In the comparison-integration mode, the subject compares adjacent digits across pairs and then combines the differences to select the larger. Algebraically, the two modes are identical. Cognitively, they are probably quite different.

A number of studies have investigated response times for the component operations of comparison and addition. If it is assumed that the empirical effects in the separate tasks also obtain within the component operations of the more complex task, then the pattern of these effects may be used a posteriori to assess the modes of processing. Studies investigating response times of subjects to select the larger of two single digits have resulted in two major empirical findings. First, Moyer and Landauer (1967) found that response time is an indirect function of the absolute difference or split between the two numbers. Consequently, the time it takes a subject to respond that 2 is greater than 1 is longer than the time to respond that 9 is greater

This project was supported in part by a grant from the General Research Board of the University of Maryland. Computing time was donated by the University of Maryland Computer Science Center. The author thanks Sidney Connor for his assistance in conducting the research. Requests for reprints should be addressed to Kent L. Norman, Department of Psychology, University of Maryland, College Park, Maryland 20742. than 1. Second, Parkman (1971) found that response time increases with the magnitude of the minimum digit for any given split. For example, response time is longer for digits 8 and 9 than for 1 and 2. These two effects have been replicated by Buckley and Gillman (1974), Sekuler, Rubin, and Armstrong (1971), and others. These findings stand as a signature of the comparison process and may be used to detect the occurrence of a comparison stage in more complex tasks.

Similarly, studies on addition by Parkman and Groen (1971) and by Restle (1970) show that response time is a function of the magnitudes of the numbers. Three empirical findings are relevant here: Response time is (1) a direct function of the magnitude of the first addend, (2) a direct function of the magnitude of the second addend, and (3) longer for an ascending order of numbers than for a descending order.

In the present experiment, stimulus sets were constructed so as to vary the magnitudes of digits entering into each potential component of processing. In addition to a simultaneous presentation of the four digits, two sequential presentation conditions investigated the effect of prior knowledge of the values of some digits on the mode of processing used. In one case, the left pair of numbers was displayed during the foreperiod; in the other case, the top number in each pair was displayed during the foreperiod. The first sequential condition should activate an integrationcomparison mode and the second should activate a comparison-integration mode.

\section{METHOD}

\section{Subjects}

Thirty students ( 15 male and 15 female) from the University of Maryland volunteered to serve as subjects. Five males and five females participated in each of three conditions. An additional three subjects were dropped from the experiment for producing more than $6.25 \%$ errors. 


\section{Material and Apparatus}

Sets of four numbers (two forming a left column and two forming a right column) were constructed from the digits 1 to 5 , with the restrictions that within any set (1) no number was repeated in either column, (2) no number occurred in both the left and right columns, and (3) the column totals were not equal. The first restriction was made in light of the finding that equal addends lead to more rapid response times than do unequal addends (Suppes \& Groen, 1967), and the second was made to eliminate cancellation of equal digits across pairs. The restrictions resulted in the following 12 sets: $23-15,15-34$, $13-24,14-25,24-35,13-25,14-35,12-34,23-45,12-35,13-45$, $12-45$, where the pair of digits on either side of the dash are the addends listed in columns. The frequency distributions for both single digits and column totals for these sets are nearly uniform. Eight orders of the numbers within each set were formed by permutating (1) the digits in the column with the smaller total, (2) the digits in the column with the larger total, and (3) the two columns. Four practice items were constructed in which all five digits appeared according to the restrictions listed.

The left and right columns were displayed on positive transparencies in separate fields of a Scientific Prototype threechannel autotachistoscope (Model GB). A vertical line separated the left and right fields. The view illumination was approximately $20 \mathrm{fL}$. Numbers were $6 \times 4 \mathrm{~mm}$ as they appeared on the slides. Numbers within a column were separated by $3 \mathrm{~mm}$ and columns were separated by $12 \mathrm{~mm}$. Columns subtended a visual angle of $3 \mathrm{deg}$. Response buttons directly beneath the binocular viewing hood were $6 \mathrm{~cm}$ apart. Response times were recorded to the nearest millisecond by a Hewlett Packard 5302A counter.

\section{Procedure}

Subjects were required to choose the column of digits having the greater sum by pressing the button corresponding to the side on which that sum appeared. In each condition a blank field illuminated for $1.50 \mathrm{sec}$ served as a ready signal. In the simultaneous condition the two columns of numbers were then illuminated until the subject pressed one of the two response buttons. At this point the fields went blank for an intertrial interval (ITI) of $3.25 \mathrm{sec}$. In the pairwise sequential presentation condition the left column then appeared for $1.75 \mathrm{sec}$, followed by the right column. The two fields remained illuminated until the subject responded, at which time they went blank for an ITI of $1.50 \mathrm{sec}$. In the memberwise sequential presentation the first digit in each column then came on for $1.75 \mathrm{sec}$, followed by the second digits. All four digits remained until the subject responded, at which time the fields went blank for an ITI of $1.50 \mathrm{sec}$. Subjects were given 4 practice trials and 96 test trials in a session lasting approximately $15 \mathrm{~min}$.

\section{RESULTS}

\section{Simultaneous Presentation}

The error rate of selecting the incorrect pair was $2.5 \%$, with $45 \%$ of the errors occurring on Set $23-15$ and $29 \%$ on Set $15-34$. The remaining $26 \%$ tended to be uniformly distributed across the rest of the sets.

Means for the 12 sets of numbers (averaged over subjects and the eight orders of numbers) are graphed in the first panel of Figure 1 as a function of split in total magnitude between the two pairs. As split increased, mean response time decreased. A planned comparison for this effect was significant $[F(5,209)=$ $39.58, \mathrm{p}<.01]$. This effect is not unlike the split effect for single digits.

When response time was considered as a function of the minimum total magnitude for each split, two effects were significant. Response time for Set 15-34 was less than that for Set 23-15 $[\mathrm{F}(1,19)=5.42$, $\mathrm{p}<.05$ ] and response time for Set $12-34$ was less than that for Set $23-45[F(1,19)=8.20, p<.01]$. The first is opposite that observed for single digits. Typically, as the magnitude of digits increases for a fixed split, response time increases; but in the first effect above, response time was greater for the set having the smaller total magnitude. Furthermore, this effect is contrary to response times observed for the addition of two digits. Typically, addition of smaller numbers tends to be faster than addition of larger numbers (Parkman \& Groen, 1971). The difference between 12-34 and $23-45$ is in line with the addition and comparison results observed for single digits.

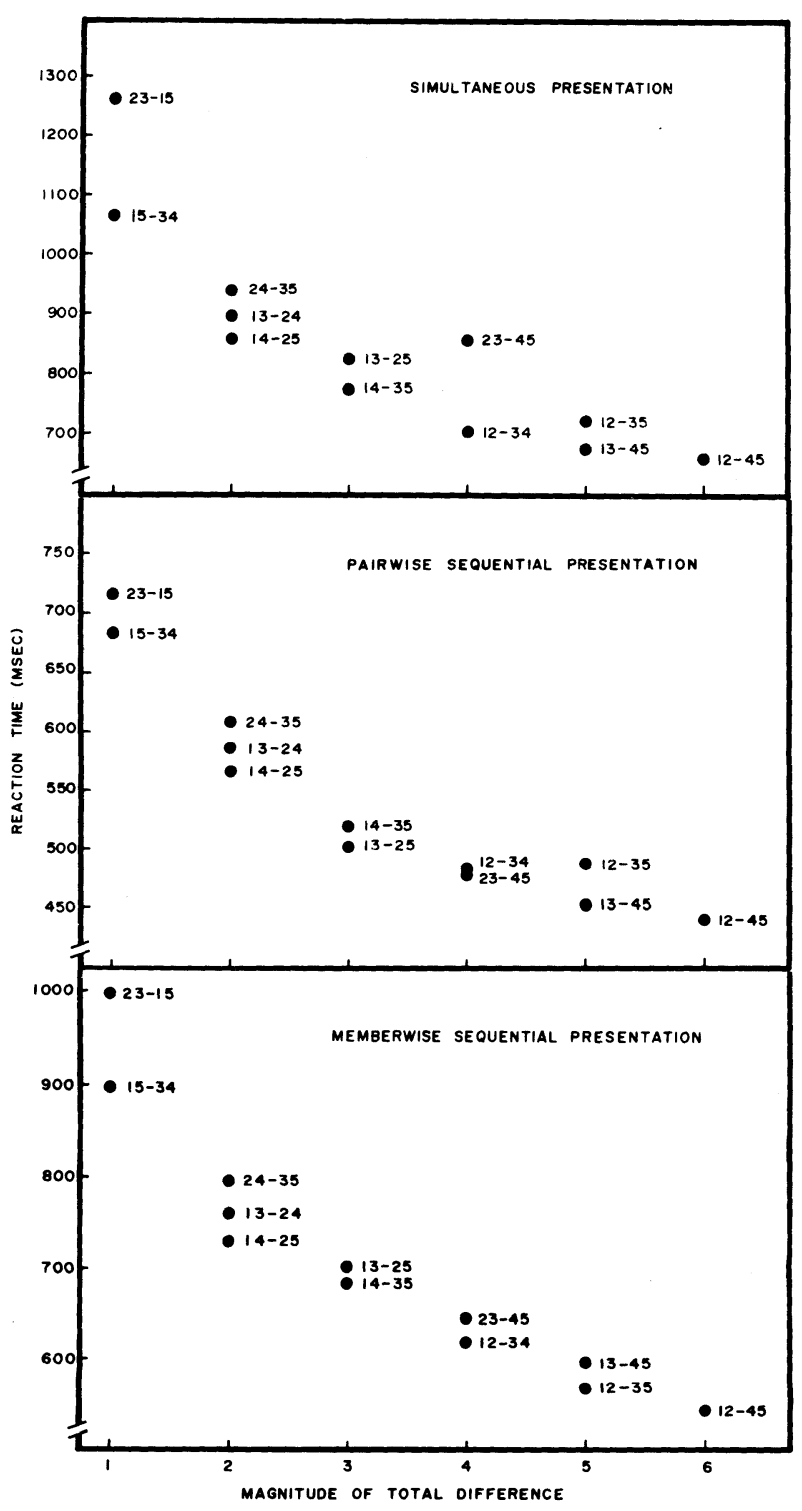

Figure 1. Mean response time for each number set as a function of the total difference between the pairs (split) in the three presentation conditions. 
Examination of the effects of order for each set revealed significant effects for 5 of the 12 sets. Four main effects indicated faster response times when the order of the digits in one column was descending. Three of the effects occurred in columns having the larger total $(13-24,14-25,24-25, p<.01$ in each case); the remaining effect occurred in the column having the smaller total $(13-24, \mathrm{p}<.05)$. This type of order effect was reported by Parkman and Groen (1971) and by Suppes and Groen (1967) in tasks requiring subjects to compute the sum of two numbers.

Two interactions between different orders occurred. One was between the two within-column orders in Set 14-35 $(p<.05)$. A crossover was obtained such that the descending order in the smaller column facilitated responding when the order was also descending in the larger column, but was inhibitory when the order in the larger was ascending. Finally, for Set $13-45$ an interaction between the order of the larger column and the order between columns occurred $(p<.05)$. This effect was small and inconsistent with other effects.

\section{Pairwise Sequential Presentation}

Again the error rate was low (2.0\%), with $29 \%$ of the errors occurring on Set $23-15$ and $21 \%$ occurring on Set $15-34$. The remaining $50 \%$ tended to be uniformly distributed across the remaining sets. Means for the 12 sets of numbers are graphed in the second panel of Figure 1 as a function of split in total magnitude. The split effect was of a lesser magnitude than that for the simultaneous presentation condition $[F(5,209)=36.46$, $p<.01]$. No significant differences occurred for response times between stimulus sets having the same split in total magnitude.

Order effects were significant for 7 of the 12 sets. In six main effects, the order of presentation of the pairs had an effect. In Sets 23-15, 14-25, and 13-24, response time was faster when the total of the first pair equaled one of the numbers in the second pair $(p<.01$ in each case). This suggests a strategy of cancellation. A main effect for Set 15-34 indicated that response time was faster when the smaller pair was shown first $(p<.05)$, as was true of the previous three main effects. Order effects for Sets $23-45$ and 12-45 were probably due to an acquired foreknowledge of which alternative would be larger or smaller in the context of the present experiment $(\mathrm{p}<.05)$.

Main effects obtained in Sets 23-45 and 15-34 that were due to the order of 45 in the first case and 15 in the second $(\mathrm{p}<.01$ in each case). As in the simultaneous presentation condition, subjects took longer to integrate numbers in an ascending than in a descending order. A significant interaction obtained between the order of Pair 24 and the order of presentation of columns in Set 13-24 $(\mathrm{p}<.01)$. Response time was faster for the descending order 42 when this pair was presented second. A similar interaction obtained between the order of Pair 25 and the order of columns in Set 13-25 $(\mathrm{p}<.01)$.

\section{Memberwise Sequential Presentation}

As in the previous two conditions, error rate was low $(2.7 \%$ ), with $35 \%$ of the errors occurring on Set $23-15,32 \%$ occurring on Set 15-34, and the remaining $33 \%$ distributed across the remaining sets. Means for the number sets are graphed in the third panel of Figure 1 as a function of the split in total magnitude. The split effect was about intermediate between the two previous conditions. The planned comparison for this effect was significant $[F(5,209)=$ $68.52, \mathrm{p}<.01]$. There were two significant differences due to magnitude for sets having the same split. The response time for Set 15-34 was less than for 23-15 $[F(1,19)=15.25, \mathrm{p}<.01]$ and response time varied among Sets $14-25,13-24$, and $24-35[F(1,19)=3.66$, $p<.01]$. Neither of the effects is in line with response times for adding the members of each pair and comparing totals.

For this condition, eight main effects occurred in which there was a faster response time when the smaller number (in either the left or right pair) was presented second. For $15-34,24-35,14-25,13-24$, and 12-35, this was true for the pair having the smaller total $(\mathrm{p}<.05)$. For 13-24, 14-35, and 13-45, the effect obtained in the pair having the greater total $(p<.05)$. For most of the number sets, presenting the smaller second resulted in a larger split between the bottom two digits. This served to facilitate the comparison of adjacent digits.

In three interactions there was an advantage when the difference between the first members of the pairs (split of adjacent digits during the foreperiod) and the difference between the second members (split of adjacent digits during response time) were in the same direction. This occurred in Sets 13-24, 14-25, and $13-25(\mathrm{p}<.05$ in each case). The interactions indicate an increased response time when the subject was given information that the direction of the difference was one way and then given contrary information that the difference went in the reverse direction. One remaining interaction occurred for Set $13-45(\mathrm{p}<.01)$. It appeared that when the top and bottom adjacent splits were similar in size (e.g., 2 and 3 ), response times were faster than when they were different (e.g., 1 and 4).

\section{DISCUSSION}

Numeric processing typically involves both integration operations (e.g., addition or multiplication) and comparison operations (e.g., same-different or larger-smaller judgments). Processing models may represent these operations as stages and, as such, attempt to show that they are separate noninteracting processes (Sternberg, 1969). In the present task, two complimentary processing models may be considered. In the integration-comparison model, subjects add two columns separately to arrive at their respective totals. Then the two totals are compared as single numbers to yield the appropriate response. In the comparison-integration model, subjects compare single digits across the columns to arrive at inequalities or differences. Then these are integrated to yield the appropriate response.

\section{Integration-Comparison Model}

In the simultaneous presentation condition, the integration 
process for each column should follow the same course as for single digits. The order effect in which descending columns are summed faster than ascending was obtained, but one of the differences between response times for a fixed split (Set 23-15 vs. Set 15-34) ran contrary to the finding that it takes longer to add larger numbers than smaller numbers. Similarly, the comparison process should follow the same temporal course as with single digits. The split effect for total magnitude was clearly obtained, but again the difference between response times for Sets 23-15 and 15-34 ran contrary to the finding that comparison is faster for a smaller than for a larger minimum.

In the pairwise sequential presentation condition, subjects probably adopted an integration-comparison strategy, since more than enough time was supplied during the foreperiod for adding the left column. Observed order effects in the right column are consistent with the integration stage, and the observed split effect is consistent with the comparison stage. However, the effects observed for order of presentation of the columns suggest that, when the total of the first column is equal to one of the single digits in the second column, subjects bypass the integration stage on the right column. Aside from this last finding, the results of the pairwise sequential presentation condition are more in line with the integrationcomparison model than are the results of the simultaneous presentation condition.

\section{Comparison-Integration Model}

Assume that subjects using the comparison-integration strategy make comparisons only between corresponding digits in the two columns and that the comparisons follow the split and minimum magnitude effects. In integrating these comparisons and translating their effects onto the observed response times, one expects that the larger the split, or the smaller the minimum for a fixed split in the right direction (i.e., same direction as total magnitude), the faster the response time. Conversely, one expects that the larger the split, or the smaller the minimum for a fixed split in the wrong direction, the slower the response time.

These predictions agree with the split effect observed in the simultaneous presentation condition; more interestingly, they agree with the direction of the significant differences observed between sets having the same split. Table 1 illustrates this effect for the difference between Sets 23-15 and 15-34 and between Sets $23-45$ and 12-34. Only two orders of the sets need to be shown. Comparisons of corresponding numbers reveal the same splits but different minimums. In Set 23-15 the comparison of 2 with 1 has a minimum less than the comparison of 5 with 4 in Set 51-43. Since both are in the wrong direction, the one with the larger minimum (51-43) should have the faster response time. Furthermore, in Set 23-15 the comparison of 3 with 5 has a minimum greater than the comparison of 1 with 3 in Set 51-43. Since both are in the right direction, the one with the smaller minimum (again 51-43) should have the faster response time. A similar analysis of Sets 23-45 and 12-34 conforms to the observation that the response time was faster for Set 12-34. The comparisonintegration model, however, does not account for the observed main effects due to the within-column ascending vs. descending order that was clearly consistent with the addition stage in the integration-comparison model. It does account for the interaction due to orders for Set 14-35. When comparisons of digits across columns are both in the right direction, overall response times are faster than when one comparison is in the wrong direction.

The comparison-integration model does not follow from the results in the pairwise sequential presentation condition. While the overall split effect may be accounted for, no effects were significant between sets for a given split. Furthermore, effects due to the order of the pairs were inconsistent with an integration-comparison model.
Table 1

Comparison-Integration Model Analysis for Two Effects in the Simultaneous Presentation Condition

\begin{tabular}{|c|c|c|c|c|c|c|c|c|}
\hline \multirow[b]{2}{*}{ Contrast } & \multicolumn{4}{|c|}{$\begin{array}{l}\text { Set with Slower } \\
\text { Response Time }\end{array}$} & \multicolumn{4}{|c|}{$\begin{array}{l}\text { Set with Faster } \\
\text { Response Time }\end{array}$} \\
\hline & & & Split & $\mathbf{M}$ & & & Split & M \\
\hline \multirow[b]{2}{*}{$23-15$ vs. $15-34$} & 2 & $\begin{array}{l}1 \\
5\end{array}$ & $\begin{array}{r}-1 \\
2\end{array}$ & $\begin{array}{l}1 \\
3\end{array}$ & 5 & $\begin{array}{l}4 \\
3\end{array}$ & -1 & $\begin{array}{l}4 \\
1\end{array}$ \\
\hline & $\begin{array}{l}3 \\
2\end{array}$ & $\begin{array}{l}1 \\
5\end{array}$ & $\begin{array}{r}-2 \\
3\end{array}$ & $\begin{array}{l}1 \\
2\end{array}$ & $\begin{array}{l}5 \\
1\end{array}$ & $\begin{array}{l}3 \\
4\end{array}$ & $\begin{array}{r}-2 \\
3\end{array}$ & $\begin{array}{l}3 \\
1\end{array}$ \\
\hline \multirow{2}{*}{$23-34$ vs. $12-34$} & $\begin{array}{l}2 \\
3\end{array}$ & $\begin{array}{l}4 \\
5\end{array}$ & $\begin{array}{l}2 \\
2\end{array}$ & $\begin{array}{l}2 \\
3\end{array}$ & $\begin{array}{l}1 \\
2\end{array}$ & $\begin{array}{l}3 \\
4\end{array}$ & $\begin{array}{l}2 \\
2\end{array}$ & $\begin{array}{l}1 \\
2\end{array}$ \\
\hline & $\begin{array}{l}3 \\
2\end{array}$ & $\begin{array}{l}4 \\
5\end{array}$ & $\begin{array}{l}1 \\
3\end{array}$ & $\begin{array}{l}3 \\
2\end{array}$ & $\begin{array}{l}2 \\
1\end{array}$ & $\begin{array}{l}3 \\
4\end{array}$ & $\begin{array}{l}1 \\
3\end{array}$ & $\begin{array}{l}2 \\
1\end{array}$ \\
\hline
\end{tabular}

Note $-M=$ minimum.

Results for the memberwise sequential presentation condition conform to the comparison-integration model. The effects due to split in total magnitude may be accounted for by the component splits between adjacent digits. The significant contrast between Sets 15-34 and 23-15 can be accounted for by the analysis shown in Table 1 . A similar analysis accounts for the difference among Sets 14-25, 13-24, and 24-35. Finally, nearly all of the within-set order effects are consistent with the comparison-integration model, and three of the four interactions are consistent with only the comparison-integration model.

Subjects were able to alter their processing strategies as a function of the structure of prior information. Given a pairwise sequential presentation, subjects used an integration-comparison strategy. Given a memberwise sequential presentation, subjects used a comparison-integration strategy. However, the overall response time was substantially longer in the comparisonintegration $(712 \mathrm{msec})$ than in the integration-comparison mode $(544 \mathrm{msec})$. The results in the simultaneous presentation condition were somewhat mixed, but, on the whole, the integration-comparison strategy dominated. Apparently, the comparison operation is both more difficult and more time consuming than the integration operation for single digits.

\section{REFERENCES}

Buckley, P. B., \& Gillman, C. B. Comparisons of digits and dot patterns. Journal of Experimental Psychology, 1974, 103, 1131-1136.

Moyer, R. S., \& Landauer, T. K. Time required for judgments of numerical inequality. Nature, 1967, 215, 1519-1520.

PARKMan, J. M. Temporal aspects of digit and letter inequality judgments. Journal of Experimental Psychology, 1971, 91, 191-205.

Parkman, J. M., \& Groen, G. J. Temporal aspects of simple addition and comparison. Journal of Experimental Psychology, $1971,89,335-342$.

REstLE, F. Speed of adding and comparing numbers. Journal of Experimental Psychology, 1970, 83, 273-278.

Sekuler, R., Rubin, E., \& Armstrong, R. Processing numerical information: A choice time analysis. Journal of Experimental Psychology, 1971, 90, 75-80.

STERnBERG, S. The discovery of processing stages: Extensions of Donders' method. Acta Psychologica, 1969, 30, 276-315.

Suppes, P., \& Groen, G. J. Some counting models for first grade performance data on simple addition facts. In J. M. Scandura (Ed.), Research in mathematics and education. Washington, D.C: National Council of Teachers of Mathematics, 1967.

(Received for publication February 8, 1979.) 